\title{
Dorsal Scapular Artery Variations and Relationship to the Brachial Plexus, and a Related Thoracic Outlet Syndrome Case
}

\author{
Anne-Marie A. Verenna ${ }^{1,2}$ Daniela Alexandru ${ }^{3}$ Afshin Karimi ${ }^{4} \quad$ Justin M. Brown ${ }^{5}$ Geoffrey M. Bove ${ }^{6}$ \\ Frank J. Daly ${ }^{6}$ Anthony M. Pastore ${ }^{6}$ Helen E. Pearson ${ }^{1}$ Mary F. Barbe ${ }^{1}$
}

\footnotetext{
${ }^{1}$ Department of Anatomy and Cell Biology, Temple University School of Medicine, Philadelphia, Pennsylvania, United States

2 Delaware County Community College, Media, Pennsylvania, United States

${ }^{3}$ Department of Neurological Surgery, University of California Irvine School of Medicine, Orange, California, United States

${ }^{4}$ UC3D LABS, Del Mar, California, United States

${ }^{5}$ Division of Neurosurgery, UCSD Medical Center, San Diego, California, United States

${ }^{6}$ Department of Biomedical Sciences, University of New England College of Osteopathic Medicine, Biddeford, Maine, United States
}

Address for correspondence Mary F. Barbe, PhD, Department of Anatomy and Cell Biology, Temple University School of Medicine, 3500 North Broad St., Philadelphia, PA 19140, United States (e-mail: mary.barbe@temple.edu).

J Brachial Plex Peripher Nerve Inj 2016;11:e21-e28.

received July 14, 2015 accepted March 23, 2016
Rationale Knowledge of the relationship of the dorsal scapular artery (DSA) with the brachial plexus is limited.

Objective We report a case of a variant DSA path, and revisit DSA origins and underinvestigated relationship with the plexus in cadavers.

Methods The DSA was examined in a male patient and 106 cadavers.

Results In the case, we observed an unusual DSA compressing the lower plexus trunk, that resulted in intermittent radiating pain and paresthesia. In the cadavers, the DSA originated most commonly from the subclavian artery (71\%), with 35\% from the thyrocervical trunk. Nine sides of eight cadavers (seven females) had two DSA branches per side, with one branch from each origin. The most typical DSA path was a subclavian artery origin before passing between upper and middle brachial plexus trunks (40\% of DSAs), versus between middle and lower trunks $(23 \%)$, or inferior (4\%) or superior to the plexus (1\%). Following a thyrocervical trunk origin, the DSA passed most frequently superior to the plexus (23\%), versus between middle and lower trunks (6\%) or upper and middle trunks (4\%). Bilateral symmetry in origin and path through the brachial plexus was observed in 13 of 35 females (37\%) and 6 of 17 males (35\%), with the most common bilateral finding of a subclavian artery origin and a path between upper and middle trunks (17\%).

Conclusion Variability in the relationship between DSA and trunks of the brachial plexus has surgical and clinical implications, such as diagnosis of thoracic outlet syndrome.

- anatomical variations 


\section{Introduction}

The dorsal scapular artery (DSA) supplies the levator scapulae and rhomboid muscles, and contributes to the arterial anastomosis around the scapula. There is disagreement in the literature regarding the origin of the DSA. The origin that is most frequently cited is as a branch of the subclavian artery, and, to a lesser extent, as a branch of the thyrocervical trunk. However, reports vary, with some indicating that the DSA originates with more or less the same frequency from the thyrocervical trunk and subclavian artery (50.7 and 49.3\%, respectively $)^{1}$ and others indicating that it originates more often from the subclavian artery (70\%), ${ }^{2}$ and, more recently, more commonly from the thyrocervical trunk with a frequency of 69 to $71 \%{ }^{3-5}$ There is limited knowledge concerning the anatomical relationship of the DSA with the brachial plexus, especially when the direct origin is from the subclavian artery, ${ }^{2,3,6,7}$ even though this information is key clinically for performing surgery or nerve blocks in the posterior triangle and for diagnosis of thoracic outlet syndrome (TOS).

Arterial compression of nerves resulting in pain or dysfunction is a phenomenon recognized to occur both intracranially $^{8,9}$ and extracranially. ${ }^{10-12}$ TOS is frequently a result of anatomical variations in the region of the brachial plexus and is typically characterized by pain and paresthesias in the upper limb, accompanied occasionally by weakness. ${ }^{13-16}$ Mechanical compression of the brachial plexus by anomalous cervical ribs, fibrous bands, or hypertrophied scalene muscles is commonly implicated as the etiology of TOS. ${ }^{15,17-20}$ Upper limb symptoms can also result from mechanical compression of components of the brachial plexus by subclavian vessels or its branches as they pass through the outlet. ${ }^{13,15,17,20,21}$ The DSA is the most frequently implicated artery in this type of compression of the plexus, with a location between the middle and lower trunks of the plexus cited as the most problematic variant. ${ }^{22-24}$ Besides direct compression by subclavian vessels, ${ }^{25}$ compression of the brachial plexus by vascular branches has been reported in the literature, although only rarely. ${ }^{26}$

Our goal was to report a case of a young patient with TOS due to compression of the lower trunk of the brachial plexus by an anomalous DSA. We also examined the path of the DSA relative to the brachial plexus trunks in 158 sides of 106 cadaveric specimens and how the paths differ by DSA origin.

\section{Materials and Methods}

\section{Case Report}

We describe the case of a 17-year-old right-handed male patient who presented to the neurosurgery clinic for evaluation of right upper limb pain and paresthesia of 4-year duration. A medical history was taken in which past and current symptoms of pain, discomfort, and weakness were recorded, followed by a thorough neurological examination by a neurosurgeon. Evaluation included magnetic resonance imaging (MRI). Surgical exploration of the right brachial plexus was performed.

\section{Cadaveric Study}

Specimens were obtained from Temple University School of Medicine and University of New England College of Osteopathic Medicine. A total of 163 DSAs in 158 sides in 106 cadaveric specimens were examined ( 59 females and 47 males; more than 1 DSA was identified per side in 8 cadavers). Cadavers were embalmed and were 45 to 100 years old (mean age \pm SD $=80.30 \pm 11.98$ ). Death certificates for the 56 cadavers from Temple University indicated race as "Black" or "White." Death certificates for the 50 cadavers from University of New England were all identified as white Americans of northern European ancestry.

Cadavers were partially predissected by students enrolled in gross anatomy courses, with over half of the cadavers requiring additional dissection for this study. Only 52 cadavers could be examined bilaterally. The arteries and brachial plexus of the right and left posterior cervical triangles and axillary regions were dissected and evaluated by the authors. The DSA was identified by its course deep to the levatorrhomboid muscle groups. Cadavers were excluded if the origin of the DSA or components of the brachial plexus could not be identified or if a visible pathology or surgical/dissection intervention in the cervical, thoracic, or deltoscapular area was observed.

\section{Statistical Analysis}

Data are presented as absolute numbers and percentages. Chi-square analyses were used to determine any significant differences in data between males and female cadavers, and between left and right sides with respect to DSA origin and relationship to brachial plexus trunks.

\section{Results}

\section{Case Report of a DSA Compressing the Lower Trunk of the Brachial Plexus}

A 17-year-old right-handed male patient presented to the neurosurgery clinic for evaluation of right upper limb pain and paresthesia of 4-year duration. The patient was a baseball player and noted worsening of symptoms while playing and training for baseball season, with increasing difficulty throwing a baseball. Prior to onset of these symptoms, the patient played outfield position and threw a ball without difficulty. At the time of presentation, he was experiencing paresthesias and weakness of his grip, with symptoms exacerbated when his hand was overhead, particularly during throwing, and was only able to throw 90 to 100 feet. His paresthesias radiated down to his medial forearm and the fourth and fifth digits. The patient also noted pain medial to the right scapula, shoulder tenderness, and weakness. He had a past medical history of asthma, but was otherwise healthy and taking no medications.

On physical examination, the patient had excellent muscle bulk and bilateral symmetry. He had 5/5 motor strength in proximal muscle groups of the arms. He had a relative weakness in flexion of his right fourth and fifth digits $(-5 /$ 5), compared with the left hand. Wrist flexion on both sides demonstrated $5 / 5$ strength with ulnar deviation. Two-point discrimination results were normal $(<3 \mathrm{~mm})$ in all digits. 
Grip strength was 70 pounds on the right side, and 100 pounds on the left, despite the patient being right hand dominant. His pinch strength was 4 pounds, bilaterally. He had normal strength in the adductor pollicis muscle, and provocative maneuvers were negative for ulnar nerve entrapment, indicating no ulnar nerve neuropathy. The patient's ulnar nerve would translocate medially during elbow flexion to rest upon the medial epicondyle. Light percussion over the course of the ulnar nerve at the leading edge of the flexor carpi ulnaris produced mild paresthesia (Tinel sign), although this test only reproduced portions of the distal symptoms. All symptoms (medial scapular, shoulder, and ulnar) were reproduced with pressure over the scalene muscles in the supraclavicular fossa and while the patient held his arms overhead. The same tests produced no symptoms on the contralateral side. Electromyogram and nerve conduction studies were unremarkable; this is not unexpected, as these tests were performed in a neutral position, which did not cause symptoms in this patient.

Evaluation by MRI showed a possible vascular/fibrous band over the lower trunk of the brachial plexus distal to the first rib. - Fig. 1 illustrates this relationship using a parasagittal IDEAL T2-weighted sequence, where a linear hypointense structure is seen traversing on top of C8 (arrow). This proposed vascular/ fibrous band has an apparent connection with the adjacent subclavian artery, consistent with designation as the DSA. Combining MRI findings with patient's description of symptoms, a working diagnosis of TOS was made. The patient underwent physical therapy for 3 years, with very modest improvement of symptoms. On the basis of worsening symptoms, lack of response to physical therapy, and patient's desire to play competi-

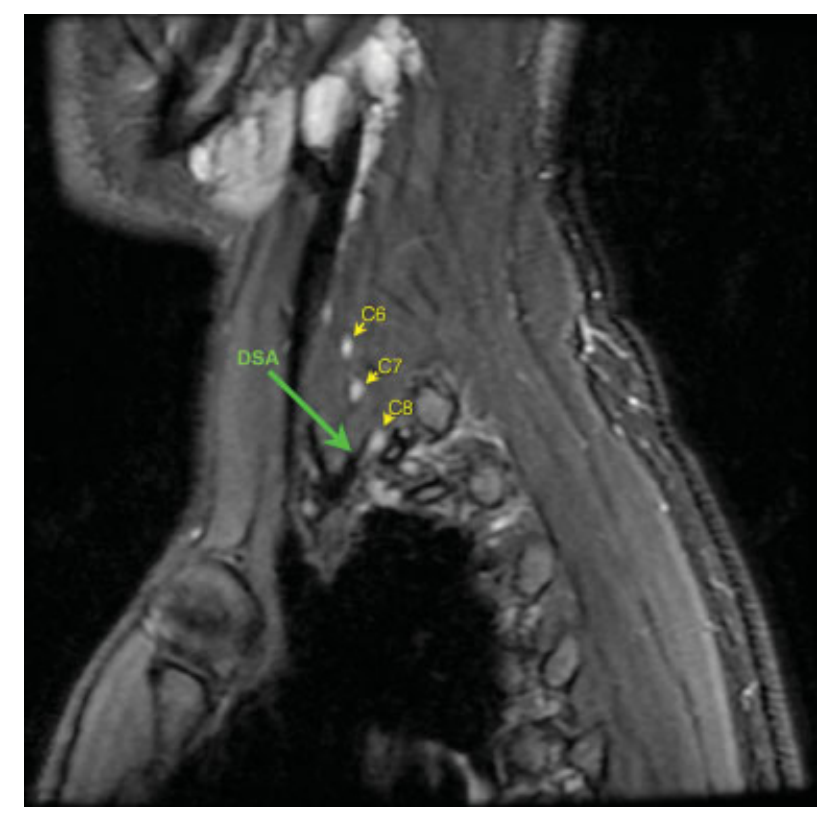

Fig. 1 Sagittal T2-weighted, water-only imaging, corresponding to the coronal T2 image. The hypointense branching structure indicated by the arrow is the putative dorsal scapular artery branching off the subclavian artery, which is impinging on the proximal C8 root. MRI acquisition was performed on GE (Waukesha, Wisconsin, United States) 3T magnet using the IDEAL sequence. Water-only images are presented. Acquisition was performed with $T_{\mathrm{E}}=60 \mathrm{~ms}$ and $T_{\mathrm{R}}=7,907 \mathrm{~ms}$. tive baseball, surgical exploration of the right brachial plexus was performed.

The brachial plexus was exposed using a transverse supraclavicular incision. A 6-cm incision was made from the lateral aspect of the sternocleidomastoid muscle to midclavicular region, parallel to and immediately above the clavicle. The platysma muscle was divided and retractors were placed. The omohyoid muscle was encountered in the scalene fat pad and retracted superiorly. The phrenic nerve was identified on the surface of the anterior scalene muscle (-Fig. 2A). The upper trunk of the brachial plexus was identified lateral to the anterior scalene muscle. The anterior scalene muscle was divided and remaining elements of the brachial plexus exposed and identified (-Fig. 2A, B). The anterior scalene muscle did not appear hypertrophic and did not appear to be the source of the patient's symptoms. Each trunk of the plexus was followed proximally to look for additional sources of impingement. Surgical exploration revealed that the C8
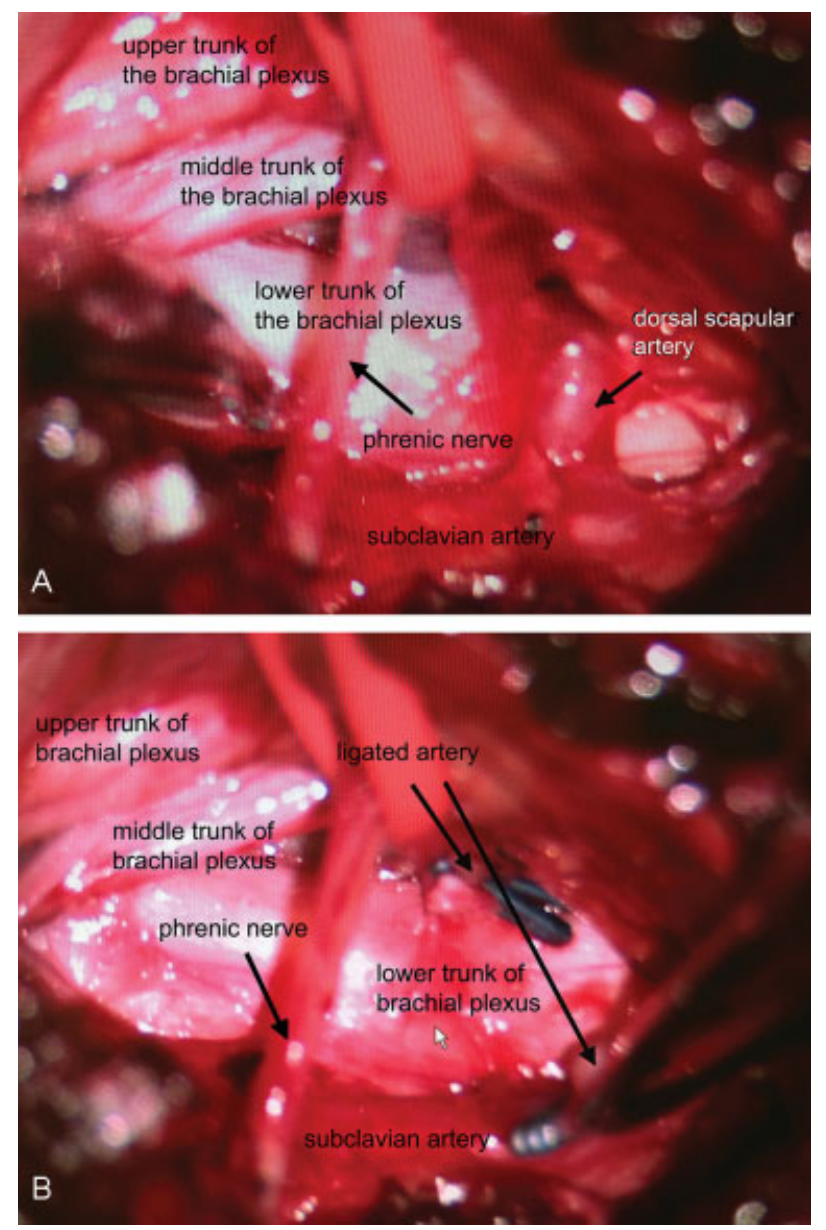

Fig. 2 Impingement of the lower trunk of the brachial plexus by the dorsal scapular artery (DSA) in a case report of a 17-year-old male. (A) The phrenic nerve (indicated with a small black arrow) and trunks of the brachial plexus were exposed and identified. The phrenic nerve is passing over the lower trunk of the brachial plexus. The $C 8$ ventral ramus contribution to the lower trunk was found to be impinged by a DSA that was $2 \mathrm{~mm}$ in diameter and that originated from the subclavian artery. This DSA crossed superior to the $\mathrm{C} 8$ ventral ramus just prior to the merger of the $\mathrm{C} 8$ and $\mathrm{T} 1$ ventral rami to form the lower trunk. (B) The artery was ligated and divided to relieve the C8 impingement. 
ventral ramus contribution to the lower trunk was impinged by a DSA that was $2 \mathrm{~mm}$ in diameter and that originated from the subclavian artery ( $\mathbf{- F i g . 2 A}$ ). This DSA crossed superior to the $\mathrm{C} 8$ ventral ramus just prior to the merger of $\mathrm{C} 8$ and $\mathrm{T} 1$ ventral rami to form the lower trunk. The DSA was tethered to the first rib, creating a physical indentation in the $C 8$ ventral ramus. The artery was ligated with titanium arterial clips and then divided to relieve the C8 impingement (-Fig. 2B). The first rib was left in situ. No further maneuvers were performed to decompress the plexus.

The patient had immediate relief of symptoms and was discharged the day after surgery. Four months postsurgery, the patient continued to be free of symptoms with full passive and active range of motion of the shoulder and cervical spine. He returned to playing baseball thereafter.

\section{Cadaveric Study}

Since the knowledge of the relationship of the DSA with the trunks and roots of the brachial plexus is under-investigated, we explored this topic, and the variability of its origin, in 158 right and left sides of 106 cadavers.

The DSA originated less from the thyrocervical trunk

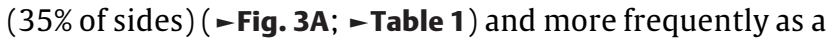
single branch of the subclavian artery (71\% of sides) (-Fig. 3B, C; - Table 1). This number is $>100 \%$ due to more than one DSA branch per side in eight cadavers (-Fig. 3D; -Table 1), as reported further later. The DSA also arose from a cervico-dorso-scapular trunk from the subclavian artery in one cadaver.

Interestingly, nine sides of eight cadavers ( $6 \%$ of sides) had two DSA branches per side, one from each origin ( -Fig. 3D; - Table 1). More female than male sides showed this finding (eight to one; - Table 1). One female cadaver had a bilateral incidence of this double branching. The size of these double branches varied, with three cadavers showing a larger branch from the thyrocervical trunk, two cadavers with small branches from each origin, and the remaining with the larger branch from the subclavian artery.
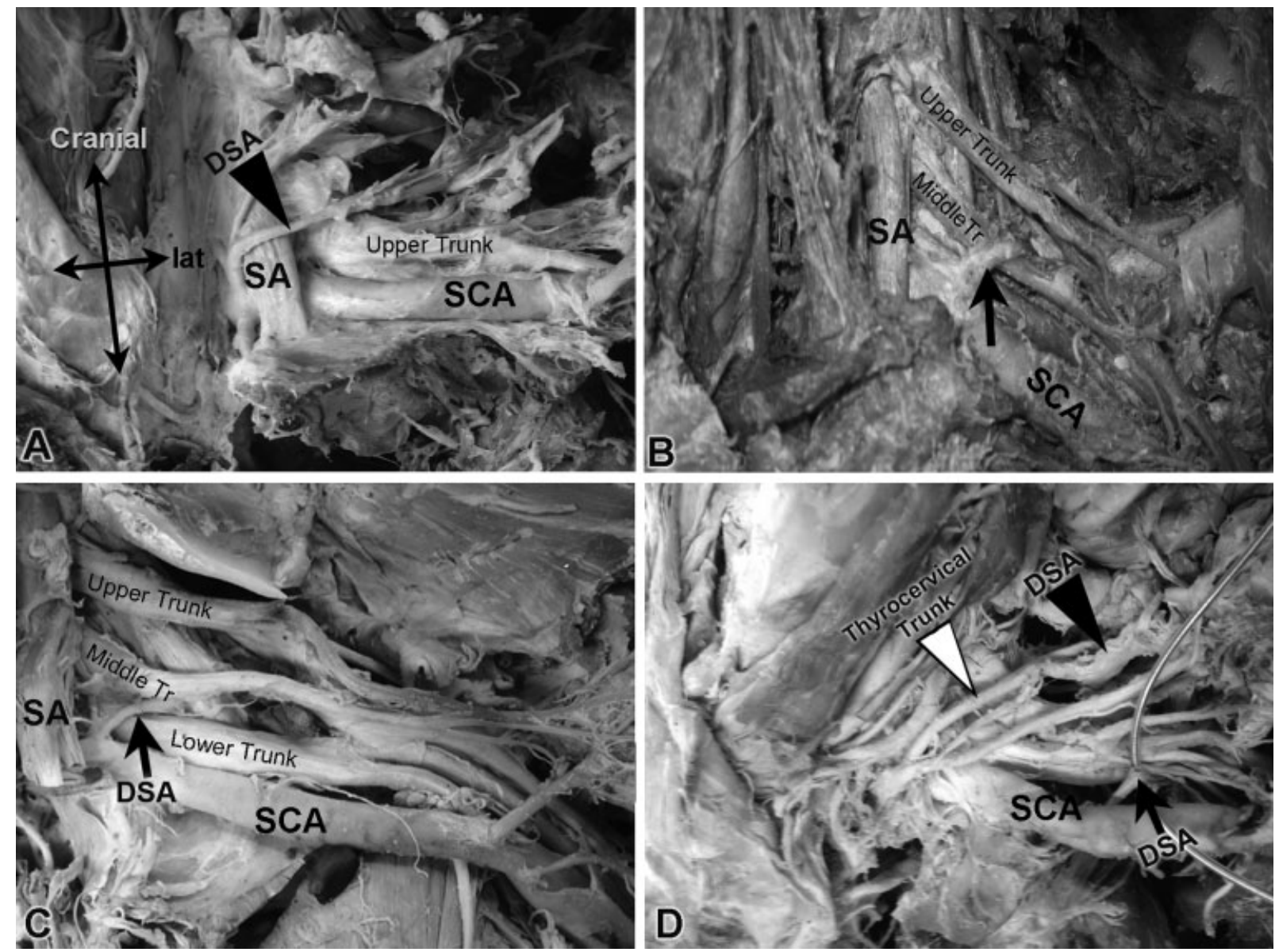

Fig. 3 Varied paths of the dorsal scapular artery (DSA) between the trunks of the brachial plexus in four different representative cadaveric specimens. (A) The DSA (arrowhead) in this cadaver originates from the thyrocervical trunk rather than from the subclavian artery (SCA). It next passes anterior to the scalenus anterior (SA) muscle (although it often passes inferior to this muscle) and superior to the brachial plexus. (B) In a different cadaver, the DSA (indicated with an arrow) originates from subclavian artery (SCA) before passing between the upper and middle trunks of the brachial plexus. Tr, trunk. (C) In another cadaver, the DSA (arrow) originates from subclavian artery before passing between the middle and lower trunks of brachial plexus. This cadaveric example is most like the case shown in - Figs. 1 and 2. (D) Two DSA branches were observed in some cadavers. In these cadavers, one of the DSA branches typically originated from the thyrocervical trunk (white arrowhead) before taking a path superior to the brachial plexus (black arrowhead). The second DSA branch (arrow) typically arose from the subclavian artery (SCA). Each DSA branch in these cadavers traveled to a similar location deep to the rhomboid muscles. 
Table 1 Origin of dorsal scapular artery and incidence (\%)

\begin{tabular}{|l|l|l|}
\hline \multirow{2}{*}{158 sides } & Thyrocervical trunk & Subclavian artery \\
\hline Males (64 sides in 47 cadavers) & 55 sides (35\%) & 112 sides (71\%) \\
\cline { 2 - 3 } & Nine sides had a branch from each origin (6\%) \\
\hline Females (94 sides in 59 cadavers) & 19 sides (30\%) & 46 sides (72\%) \\
\cline { 2 - 3 } & One side had a branch from each origin (2\%) \\
\hline
\end{tabular}

The anatomical relationship of the DSA with the brachial plexus was explored in 155 cadaveric sides (-Table 2; see also - Supplementary Table $\mathbf{S 1}$ [online-only]). The most prevalent combination of DSA origin and brachial plexus relationship was a subclavian artery origin before passing between upper and middle trunks (40\% total: $39 \%$ of males; $41 \%$ of females; - Fig. 3B; - Supplementary Table S1 [online-only]). The second most prevalent combination was an origin from the subclavian artery before passing between middle and lower trunks (23\% total: $25 \%$ of males; $21 \%$ of females; - Fig. 3C, - Supplementary Table S1 [online-only]). The third most common arrangement was a thyrocervical trunk origin before passing superior to the plexus ( $23 \%$ of total; $17 \%$ of males; 26\% of females; - Fig. 3A; - Supplementary Table S1 [online-only]). The remaining combinations were much less prevalent (-Supplementary Table S1 [online-only]).

After combining data from both sexes, chi-square analysis revealed a higher incidence for a DSA passing between upper and middle trunks than between middle and lower trunks, after an origin from the subclavian artery $(p=0.004)$. DSAs originating from the subclavian artery were more likely to pass between upper and middle trunks on the right side $(p=0.016)$, than on the left ( $p=0.108$ ). When data from both sexes and both origins were combined, $45 \%$ passed between upper and middle trunks,
$28 \%$ between middle and lower trunks, and $23 \%$ superior and $4 \%$ inferior to the plexus (-Table 2).

Chi-square analysis revealed a higher incidence of variations in females $(p=0.01)$ than males $(p=0.16)$. Females had a higher incidence for a DSA passing between upper and middle trunks (45\%), than between middle and lower trunks (25\%) or superior to the plexus (26\%), each considerably more prevalent than inferior to the plexus (3\%; - Table 2). Also in females, DSAs were more likely to pass between upper and middle trunks than between middle and lower trunks, regardless of side (chi-square test, $p=0.01$ ). Males had a higher incidence of a DSA passing between upper and middle trunks (44\%), than between middle and lower trunks (33\%), both paths being more prevalent than superior (19\%) or inferior to the plexus (6\%; - Table 2).

Bilateral symmetry in the origin and path of the DSA through the brachial plexus was observed in 6/17 males (35\%) and 13/35 females (37\%; - Table 3). One incidence of bilateral symmetry was in a female cadaver with two DSA branches per side, on both right and left sides. The most common bilateral finding was an origin for the DSA from the subclavian artery with a path between the upper and middle trunks ( $17 \%$ total: $18 \%$ of males, $17 \%$ of females). The second most common bilateral symmetry was a thyrocervical trunk

Table 2 Incidence of path of dorsal scapular artery between brachial plexus trunks from either arterial origin

\begin{tabular}{|c|c|c|c|c|c|c|c|c|c|c|c|}
\hline \multirow[t]{4}{*}{ Sex } & \multirow[t]{4}{*}{ Side } & \multicolumn{10}{|c|}{ Origin: either subclavian artery or thyrocervical trunk } \\
\hline & & \multirow{2}{*}{\multicolumn{2}{|c|}{ Total }} & \multicolumn{8}{|c|}{ Path through brachial plexus trunks } \\
\hline & & & & \multicolumn{2}{|c|}{$\mathrm{U} / \mathrm{M}$} & \multicolumn{2}{|l|}{$M / L$} & \multicolumn{2}{|l|}{ Sup } & \multicolumn{2}{|l|}{ Inf } \\
\hline & & No. & $\%$ & No. & $\%$ & No. & $\%$ & No. & $\%$ & No. & $\%$ \\
\hline \multirow[t]{3}{*}{ Female } & Right (R) & 56 & 34 & 26 & 46 & 11 & 20 & 17 & 30 & 2 & 4 \\
\hline & Left (L) & 43 & 26 & 19 & 44 & 14 & 33 & 9 & 21 & 1 & 2 \\
\hline & Both ( $R$ and $L$ ) & 99 & 61 & 45 & 45 & 25 & 25 & 26 & 26 & 3 & 3 \\
\hline \multirow[t]{3}{*}{ Male } & $R$ & 35 & 21 & 15 & 43 & 13 & 37 & 7 & 20 & 2 & 6 \\
\hline & $\mathrm{L}$ & 29 & 18 & 13 & 45 & 8 & 28 & 5 & 17 & 2 & 7 \\
\hline & Both & 64 & 39 & 28 & 44 & 21 & 33 & 12 & 19 & 4 & 6 \\
\hline \multirow[t]{3}{*}{ Total } & $\mathrm{R}$ & 91 & 56 & 41 & 45 & 24 & 26 & 24 & 26 & 4 & 4 \\
\hline & L & 72 & 44 & 32 & 44 & 22 & 31 & 14 & 19 & 3 & 4 \\
\hline & Both & 163 & 100 & 73 & 45 & 46 & 28 & 38 & 23 & 7 & 4 \\
\hline
\end{tabular}

Abbreviations: Inf, inferior to lower trunk; M/L, between middle and lower trunks; Sup, superior to upper trunk; U/M, between upper and middle trunks. 
Table 3 Incidence of bilateral DSA symmetry in male and female cadavers

\begin{tabular}{|l|l|l|l|l|l|l|l|l|}
\hline \multirow{2}{*}{$\begin{array}{l}\text { No. examined } \\
\text { bilaterally }\end{array}$} & \multicolumn{3}{|l|}{$\begin{array}{l}\text { No. of cadavers with DSA origin from } \\
\text { subclavian artery, bilaterally }(\boldsymbol{n}=\mathbf{2 1})\end{array}$} & \multicolumn{3}{l|}{$\begin{array}{l}\text { No. of cadavers with DSA origin from } \\
\text { thyrocervical trunk, bilaterally }(\boldsymbol{n}=\mathbf{7})\end{array}$} \\
\cline { 2 - 10 } & U/M & M/L & Sup & Inf & U/M & M/L & Sup & Inf \\
\hline Males $(n=17)$ & $3(18 \%)$ & $2(12 \%)$ & 0 & 0 & 0 & 0 & $1(6 \%)$ & 0 \\
\hline Females $(n=35)$ & $6^{\text {a }(17 \%)}$ & $3(9 \%)$ & 0 & 0 & 0 & 0 & $5^{\text {a }}(14 \%)$ & 0 \\
\hline Total $(n=52)$ & $9(17 \%)$ & $5(10 \%)$ & 0 & 0 & 0 & 0 & $6(12 \%)$ & 0 \\
\hline
\end{tabular}

Abbreviations: DSA, dorsal scapular artery; Inf, inferior to lower trunk; M/L, between middle and lower trunks; Sup, superior to upper trunk; U/M, between upper and middle trunks.

${ }^{a}$ One female cadaver had two branches per side, one with a subclavian origin and one with a thyrocervical origin.

origin and a path superior to the upper trunk (12\% total, $6 \%$ of males, and $14 \%$ of females) ( - Table 3 ). Bilateral symmetry of the DSA was also found for the artery of origin, with two males and five females having bilateral origins of the DSA from the subclavian artery (-Table $\mathbf{3}$ ).

\section{Discussion}

The patient case shows that a path of the DSA over the $\mathrm{C} 8$ and $\mathrm{T} 1$ roots of the brachial plexus that resulted in intermittent radiating neurologic symptoms in the subject that were exacerbated when his hand was overhead. While the brachial plexus is more typically mechanically compressed in the thoracic outlet by bone, muscle, $3,15,18,21,22,27$ or fascial structures, ${ }^{19,28}$ other important potential sources of compression are vascular structures in this region. ${ }^{24,26,27}$ There is one case report describing an abnormal cervical artery and vein complex compressing the upper trunk of the brachial plexus and causing TOS symptoms. ${ }^{22}$ Our patient case describes compression of the lower trunk of the brachial plexus by an abnormal course of the DSA. The clinical findings in our patient correlated well with lower brachial plexus compression. Surgical exploration of the brachial plexus revealed that the $\mathrm{C} 8$ ventral ramus was compressed by a DSA arising from the third portion of the subclavian artery and then passing superior to the C8 ventral ramus. The patient's symptoms fully resolved postsurgery, confirming an arterial compression of $\mathrm{C} 8$ ventral ramus as responsible for the TOS symptoms. These findings are similar to a prior anatomical study on one cadaver in which a DSA compromised the T1 ventral root. ${ }^{24}$ The authors hypothesized that in some cases of TOS clinical symptoms might occur when a DSA originates from the third portion of the subclavian artery. ${ }^{24}$ Our case confirms this hypothesis. Thus, the possibility of a vascular compression of the brachial plexus should be considered in differential diagnoses of TOS symptoms.

Previous studies have also reported that variant patterns of the DSA are partially responsible for the failure of a brachial plexus block. Kinjo and Frankel described an inability to provide anesthesia in a patient due to the possibility of piercing the DSA. ${ }^{29}$ Based on ultrasound imaging, it has been suggested that $90 \%$ of patients undergoing subclavian block are at risk for vessel puncture. ${ }^{30}$ The findings from our anatomical study of $>40 \%$ DSA passage through upper and middle trunks and $>25 \%$ passage between middle and lower trunks are consistent with the findings using ultrasound. ${ }^{30}$ This relationship is important to clinicians since DSA puncture could lead to complications, for example, incomplete analgesia and hematoma formation. ${ }^{29,30}$

It is known that variability occurs in the origin of the DSA. We found in our study of 158 sides in 106 cadavers that there is a 6\% frequency for two DSA branches per side, with one DSA branch originating from the thyrocervical trunk and the other from the subclavian artery. This finding has been previously reported in one study, although it did not report the frequency of these findings. ${ }^{31}$ Our other results are in fair agreement with several other cadaveric studies identifying the DSA as a direct branch of the subclavian artery in 67 to $75 \%$ of cadavers and from the thyrocervical trunk in the remaining cadavers $(\sim 30 \%)^{2,31-37}$ That said, a recent study found that the thyrocervical trunk is the primary origin of the DSA in $69 \%$ of cadaveric cases in which a Thai population was examined, ${ }^{3}$ matching findings from two studies examining Japanese populations, ${ }^{4,38}$ suggesting that Asians may have a higher incidence of a thyrocervical trunk origin for the DSA. ${ }^{3}$ This may be one underlying reason for the differences between our study and these latter three studies, as our cadaveric specimens were of black or white ancestry. Thus, the origin of DSA should be considered variable and perhaps dependent on ancestry, and the possibility of two DSA branches per side be considered during surgical procedures in the posterior triangle.

The prevalence and location of DSA-brachial plexus relationship is highly variable and understudied. ${ }^{3}$ The DSA often acts as a nutrient artery to brachial plexus trunks and cords, ${ }^{39}$ supporting our findings of its path through the plexus in $74 \%$ of cadavers, rather than superior or inferior to it. The most frequent variation for the DSA observed in our study was a subclavian origin before passing between upper and middle trunks. We observed that $45 \%$ of DSAs, from either origin, passed between the upper and middle trunks. A similar DSA path between upper and middle trunks has been reported in $25,263.2,{ }^{3}$ or 47 to $89 \%$ of cadavers, depending on if the DSA arose from the second or third parts of the subclavian artery. ${ }^{31}$ Concerning the DSA path between middle and lower trunks, we observed this relationship in $28 \%$ of DSAs. A similar DSA path between middle and lower trunks has been reported in $18.4,{ }^{3} 11,{ }^{31}$ and $41 \%$ of cadavers. ${ }^{2}$ The study of 
Chaijaroonkhanarak et al identified few incidences (6.6\%) of the DSA passing inferior to the plexus, ${ }^{3}$ as did we (4\%). However, their study identified the occurrence of the DSA passing superior to the plexus more frequently (7.9\%) than in our current study (1\%). The DSA has rarely been reported as passing between the roots of $\mathrm{C} 8$ and $\mathrm{T} 1$. We observed this only in the patient case in this study. Two prior cadaveric studies reported an incidence of 2.6 to $3.6 \% .^{2,3}$ We also report bilateral symmetry in both DSA origin and path through the brachial plexus in 13/35 females (37\%) and 6/17 males (35\%), indicating that for patients undergoing procedures in both posterior triangles, findings from one side should inform the other.

The relationship of DSA with the brachial plexus trunks and roots (ventral rami) after origin from the transverse cervical artery or thyrocervical trunk is also understudied. After an origin from the transverse cervical artery, the DSA has been reported to pass superior to the brachial plexus rather than through it., ${ }^{2,3}$ Although the incidence was not indicated in a review by Klaassen et al, Fig. 2 of that review shows a branch that appears to be the DSA originating from the thyrocervical trunk and passing between upper and middle trunks ${ }^{17}$ (observed in $4 \%$ of DSAs in our study).

The difference in frequency of DSA origin from the subclavian artery and the relationship with the brachial plexus between left and right sides may be the result of differences during embryological development. The right subclavian artery arises from three different structures during embryological development: the right fourth aortic arch (proximal portion), the right dorsal aorta (middle portion), and the right seventh segmental artery (distal portion). ${ }^{40}$ The left subclavian artery develops entirely from the left seventh segmental artery. ${ }^{40}$ These differences in embryological development may affect the gross anatomical location of the left and right subclavian arteries and partially explain why, in both sexes, a DSA with a subclavian origin was more likely to pass between the upper and middle trunks of the plexus on the right side.

\section{Conclusion}

The DSA has rarely been reported as passing between the roots of $\mathrm{C} 8$ and $\mathrm{T} 1$, a finding observed in our patient case presentation. This is the first literature report of a variant DSA compressing the lower trunk of the brachial plexus and leading to TOS with neurologic symptoms. In the cadaveric studies, we observed a $6 \%$ frequency for two DSA branches per side and a reasonable incidence of bilateral symmetry in origin and path of the DSA through the brachial plexus ( $37 \%$ of females and $35 \%$ of males). We suggest that in cases of upper extremity radiating pain, paresthesia, or weakness with obvious proximal etiology, the possibility of a vascular compression of the brachial plexus should be considered. Imaging using MRI (used here), ultrasound, or angiography can aid in this diagnosis ${ }^{4,41}$ and may hold similar utility to the currently accepted screening for cervical ribs in this population. Additionally, these anatomical variations should be considered when performing procedures such as a brachial plexus block.

\section{Summary}

Variability in the relationship between DSA and trunks of the brachial plexus has surgical and clinical implications, such as diagnosis of TOS.

\section{Conflict of Interest}

The authors have no conflicts of interests to declare.

\section{Acknowledgments}

We would like to acknowledge the support of the Department of Anatomy and Cell Biology, Temple University School of Medicine, and Department of Biomedical Sciences of University of New England College of Osteopathic Medicine, which allowed access to the cadavers used in this study. We would also like to thank the individuals who donate their bodies for the advancement of education and research.

\section{References}

1 Thomson A. Second annual report of the Committee of Collective Investigation of the Anatomical Society of Great Britain and Ireland for the Year 1890-91. J Anat Physiol 1891;26(Pt 1):76-93

2 Huelke DF. A study of the transverse cervical and dorsal scapular arteries. Anat Rec 1958;132(3):233-245

3 Chaijaroonkhanarak W, Kunatippapong N, Ratanasuwan S, et al. Origin of the dorsal scapular artery and its relation to the brachial plexus in Thais. Anat Sci Int 2014;89(2):65-70

4 Murata H, Sakai A, Hadzic A, Sumikawa K. The presence of transverse cervical and dorsal scapular arteries at three ultrasound probe positions commonly used in supraclavicular brachial plexus blockade. Anesth Analg 2012;115(2):470-473

5 Read WT, Trotter M. The origins of transverse cervical and of transverse scapular arteries in American Whites and Negroes. Am J Phys Anthropol 1941;28(2):239-247

6 Huelke DF. The dorsal scapular artery-a proposed term for the artery to the rhomboid muscles. Anat Rec 1962;142:57-61

7 Weiglein AH, Moriggl B, Schalk C, Künzel KH, Müller U. Arteries in the posterior cervical triangle in man. Clin Anat 2005;18(8):553-557

8 Kandan SR, Khan S, Jeyaretna DS, Lhatoo S, Patel NK, Coakham HB. Neuralgia of the glossopharyngeal and vagal nerves: long-term outcome following surgical treatment and literature review. $\mathrm{Br} \mathrm{J}$ Neurosurg 2010;24(4):441-446

9 Sarsam Z, Garcia-Fiñana M, Nurmikko TJ, Varma TR, Eldridge P. The long-term outcome of microvascular decompression for trigeminal neuralgia. Br J Neurosurg 2010;24(1):18-25

10 Kaufman MR, Willekes LJ, Elkwood AI, et al. Diaphragm paralysis caused by transverse cervical artery compression of the phrenic nerve: the Red Cross syndrome. Clin Neurol Neurosurg 2012; 114(5):502-505

11 Trimble C, Reeves A, Pare L, Tsai F. Vertebral artery anomaly causing C2 suboccipital neuralgia, relieved by neurovascular decompression. J Neuroimaging 2013;23(3):421-424

12 Tubbs RS, Smyth MD, Salter G, Oakes WJ. Anomalous traversement of the suprascapular artery through the suprascapular notch: a 
possible mechanism for undiagnosed shoulder pain? Med Sci Monit 2003;9(3):BR116-BR119

13 Brantigan CO, Roos DB. Etiology of neurogenic thoracic outlet syndrome. Hand Clin 2004;20(1):17-22

14 Sanders RJ, Hammond SL, Rao NM. Diagnosis of thoracic outlet syndrome. J Vasc Surg 2007;46(3):601-604

15 Sanders RJ, Hammond SL, Rao NM. Thoracic outlet syndrome: a review. Neurologist 2008;14(6):365-373

16 Sanders RJ, Jackson CG, Banchero N, Pearce WH. Scalene muscle abnormalities in traumatic thoracic outlet syndrome. Am J Surg 1990;159(2):231-236

17 Klaassen Z, Sorenson E, Tubbs RS, et al. Thoracic outlet syndrome: a neurological and vascular disorder. Clin Anat 2014;27(5):724-732

18 Freischlag J, Orion K. Understanding thoracic outlet syndrome. Scientifica (Cairo) 2014;2014:248163

19 Ranney D. Thoracic outlet: an anatomical redefinition that makes clinical sense. Clin Anat 1996;9(1):50-52

20 Sanders RJ. Thoracic outlet syndrome. J Neurosurg Spine 2008; 8(5):497, author reply 497-498

21 Peet RM, Henriksen JD, Anderson TP, Martin GM. Thoracic-outlet syndrome: evaluation of a therapeutic exercise program. Proc Staff Meet Mayo Clin 1956;31(9):281-287

22 Chloros GD, Crosby N, Apel P, Li Z. Anomalous vasculature as direct cause of upper brachial plexus thoracic outlet syndrome. Joint Bone Spine 2009;76(1):92-94

23 Roos DB. Congenital anomalies associated with thoracic outlet syndrome. Anatomy, symptoms, diagnosis, and treatment. Am J Surg 1976;132(6):771-778

24 Tubbs RS, Tyler-Kabara EC, Salter EG, Sheetz J, Zehren SJ, Oakes WJ. Additional vascular compression of the brachial plexus in a cadaver with a cervical rib: case illustration. Surg Radiol Anat 2006;28(1):112-113

25 Ozçakar L, Kaymak B, Turan S, Akal M, Enön S, Okuyan H. Thoracic outlet syndrome, Paget-Schroetter syndrome and aberrant subclavian artery in a young man. Joint Bone Spine 2006;73(4):469-471

26 Gabriel EM, Friedman AH. Brachial plexus compression by venous aneurysms. Case illustration. J Neurosurg 1997;86(2):311

27 Duthel R, Tudor C, Motuo-Fotso MJ, Brunon J. Cervical root compression by a loop of the vertebral artery: case report. Neurosurgery 1994;35(1):140-142
28 Roos DB. Transaxillary approach for first rib resection to relieve thoracic outlet syndrome. Ann Surg 1966;163(3): 354-358

29 Kinjo S, Frankel A. Failure of supraclavicular block under ultrasound guidance: clinical relevance of anatomical variation of cervical vessels. J Anesth 2012;26(1):100-102

30 Nambyiah P, Umbarje K, Amir R, Parikh M, Oosthuysen SA. Sonographic assessment of arterial frequency and distribution within the brachial plexus: a comparison with the cadaveric record. Anaesthesia 2011;66(10):931-935

31 Reiner A, Kasser R. Relative frequency of a subclavian vs. a transverse cervical origin for the dorsal scapular artery in humans. Anat Rec 1996;244(2):265-268

32 Lischka MF, Krammer EB, Rath T, Riedl M, Ellböck E. The human thyrocervical trunk: configuration and variability reinvestigated. Anat Embryol (Berl) 1982;163(4):389-401

33 Faller A. Statistische untersuchungen über Ursprung und Kaliber der Arteria transversa colli beim Menschen. Untersuchung an 134 Halshälften. Arch Kreisl Forsch 1952;18:161-167

34 Reichert P. Der Truncus thyreocervicalis: Untersuchungen an 150 Halshälften. Bull Soc Fribourg Science Naturell 1952;42:149-181

35 Lippert H, Pabst R. Arterial Variations in Man: Classification and Frequency. Munich: J. F. Bergmann; 1985

36 Bergman RA, Afifi AK, Miyauchi R. Illustrated encyclopedia of human anatomic variation. In: D'Alessandro MP, Bergman RA, curators. Anatomical Atlases. 1988. Available at: http://www. anatomyatlases.org/AnatomicVariants/AnatomyHP.shtml

37 Standring S. Gray's Anatomy: The Anatomical Basis of Clinical Practice. Canada: Elsevier; 2008

38 Adachi B. Das Arteriensystem der Japaner. 2nd ed. Kyoto, Kaiserlich-japanische Universität zu Kyoto, in kommission bei "Maruzen Co." 1928.

39 Levy SM, Taylor GI, Baudet J, et al. Angiosomes of the brachial plexus: an anatomical study. Plast Reconstr Surg 2003;112(7): 1799-1806

40 Kau T, Sinzig M, Gasser J, et al. Aortic development and anomalies. Semin Intervent Radiol 2007;24(2):141-152

41 Stapleton C, Herrington L, George K. Sonographic evaluation of the subclavian artery during thoracic outlet syndrome shoulder manoeuvres. Man Ther 2009;14(1):19-27 